\title{
A New Technique to Solve the Instant Insanity Problem
}

HweeJung Kim, Salar Alsardary,Julie George

Department of Pharmacy Administration, University of the Sciences, Philadelphia, PA 19104

hkim1233@mail.usciences.edu

Department of Mathematics, Physics and Statistics, University of the Sciences, Philadelphia, PA 19104

s.alsard@usciences.edu

Department of Mathematics, Physics and Statistics, University of the Sciences, Philadelphia, PA 19104

juliegeorge90@gmail.com

\section{ABSTRACT}

Instant Insanity [1] consists of four cubes, each of whose six faces are colored with one of the four colors: red, blue, white, and green. The object is to stack the cubes in such a way that each of the four colors appears on each side of the resulting column. See figure 1 below [2]. Traditionally, this could be solved using graph theory. However, in this article, we introduce a new technique to solve the problem without using graph theory. We also used a Perl programming language to implement the new approach for the Instant Insanity.

\section{Indexingterms/Keywords}

Mathematical Puzzles

\section{AcademicDisciplineAndSub-Disciplines}

Graph Theory

\section{SUBJECT CLASSIFICATION}

05

\section{TYPE (METHOD/APPROACH)}

Mathematical Puzzles

\section{Council for InnovativResearch}

Peer Review Research Publishing System

\section{Journal: JOURNAL OF ADVANCES IN MATHEMATICS}

Vol. 11, No. 10

www.cirworld.com, editor@cirworld.com 


\section{INTRODUCTION}

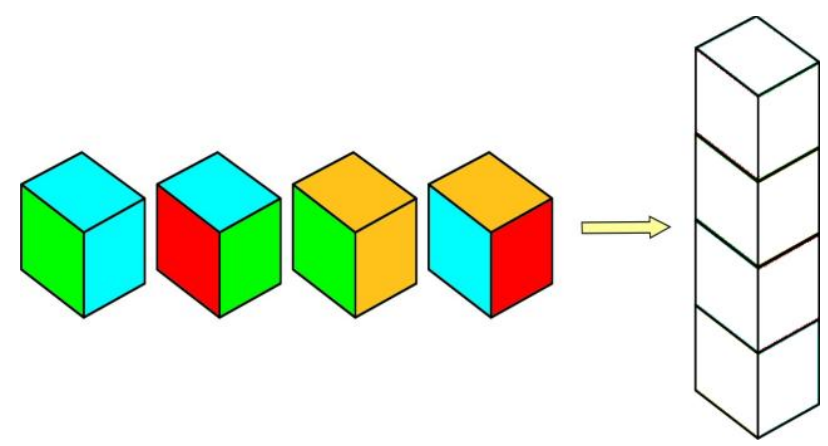

Fig 1. Visual representation of Instant Insanity Problem

This problem is practically impossible to solve by means of trial and error, since there are 41,472 arrangements possible in total. Each cube has 24 arrangements possible therefore there will be 244possiblearrangements (orderofthecubedoesnotmatter). However, notall 24 arrangementswillcountbecausethereareduplicatesolutions. Anyofthesolutionwillappear 8 times. Thereforethetotalwillbe244 / $8=41472$.

1

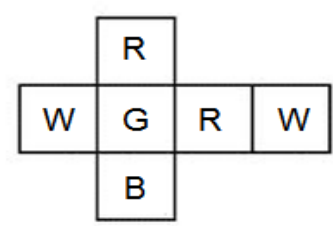

2

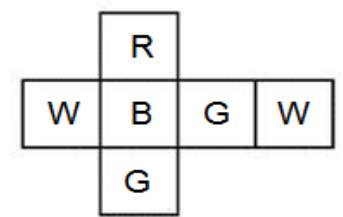

3

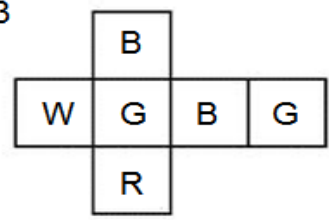

4

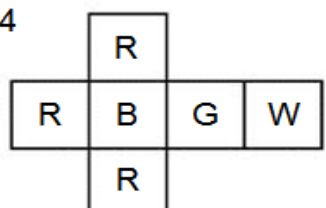

Fig 2. Opened \&flattened representation

\section{METHOD}

In this example, to illustrate the method we have in mind, we first need to imagine that the cube has been opened and flattened. We have four different cubes colored as above (figure 2). There are fivesteps to complete the stack that satisfies the conditions for Instant Insanity.

\section{STEP 1}

Count the total number of each color among the four cubes:

Red: 7

Blue: 5

Green: 6

White: 6

\section{STEP 2}




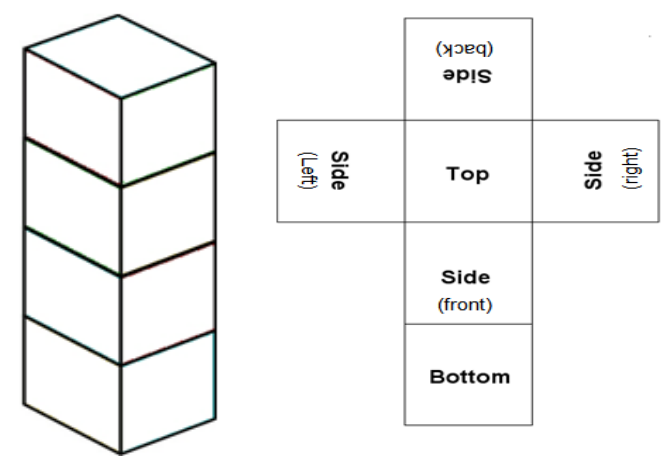

Fig 3. The schematics for the complete stack and individual cube

As in figure 3, since we want to complete the stack while each of the four colors appear on each side of the resulting column, we can draw a conclusion that we need a total of:

4 reds, 4 blues, 4 greens, and 4 whites on the column sides.

Now, we count the colors to be hidden by subtracting 4 from the total number of each color.

For instance, there are 7 reds in total. Among those 7 reds, we only need 4 of them to be placed on the column sides. Thus the remaining 3 reds must be hidden by placing them either on the top or the bottom of the cube.

Therefore, by applying the same logic to the rest, the top and the bottom must consist of:

3 Reds $(7-4=3)$

1 Blues $(5-4=1)$

2 Greens $(6-4=2)$

2 Whites $(6-4=2)$

\section{The Color Pair Concept}
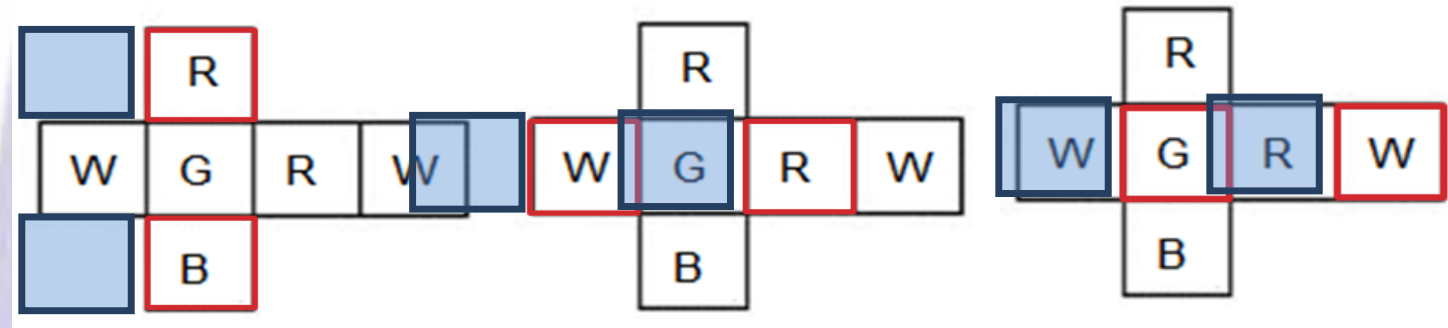

Fig 4. The top and bottom color pairs for cube 1

We can notice in figure 4 that if red is designated as the top, then blue is assigned to be the bottom - red and blue are paired (R-B).

Likewise, there are two other possible pairs that exist.

White \& Red (W-R), Green \& White (G-W)

These top and bottom color pairs are our primary focus; once top and bottom pair is selected, the colors on the sides are automatically fixed.

For instance, if we decide R-B to be the top and bottom pair, the remaining $W, G, R$, and $W$ will be placed on the sides.

\section{STEP 3}

Now we make table 1 below that consist of the top and the bottom pairs for each cube which fulfill the designated values produced in step 2: 


\begin{tabular}{|c|c|c|c|c|c|c|}
\hline & T-B Pairs & arrangement 1 & arrangement 2 & arrangement 3 & arrangement 4 & arrangement 5 \\
\hline \multirow{3}{*}{ Cube 1} & $R-B$ & $\sqrt{ }$ & & & & \\
\hline & $W-R$ & & v & V & & \\
\hline & $G-W$ & & & & $V$ & v \\
\hline \multirow{3}{*}{ Cube 2} & $R-G$ & v & | & 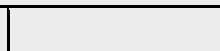 & V & \\
\hline & $W-G$ & & v & & & v \\
\hline & $W-B$ & & 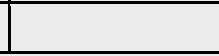 & V & & \\
\hline \multirow{3}{*}{ Cube 3} & $R-B$ & . & v & . & | & 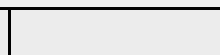 \\
\hline & $W-B$ & v & & 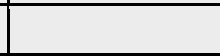 & $\mathrm{V}$ & \\
\hline & $G-G$ & | & & $\sqrt{ }$ & . & V \\
\hline \multirow{3}{*}{ Cube 4} & $R-R$ & 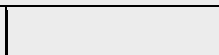 & 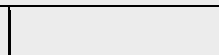 & v & v & V \\
\hline & $R-G$ & V & V & & & \\
\hline & $W-B$ & & & & & \\
\hline
\end{tabular}

Table 1. Possible arrangements based on top and bottom pairs

Note: each arrangement must satisfy theconditions from STEP 2:hiding 3 reds, 1 blue, 2 green, and 2 whites in total

\section{STEP 4}

Using the top \& bottom arrangements from step 3, we construct the front-back-left-right color in table 2 below.

\begin{tabular}{|c|c|c|c|c|c|c|c|c|}
\hline & \multicolumn{4}{|c|}{ Arrangement 1} & \multicolumn{4}{|c|}{ Arrangement 2} \\
\hline & Front & Back & Left & Right & Front & Back & Left & Right \\
\hline Cube 1 & Green & White & White & Red & Green & White & Blue & Red \\
\hline Cube 2 & Blue & White & White & Green & Blue & White & Green & Red \\
\hline Cube 3 & Green & Green & Red & Blue & Green & Green & Blue & White \\
\hline \multirow[t]{3}{*}{ Cube 4} & Blue & White & Red & Red & Blue & White & Red & Red \\
\hline & \multicolumn{4}{|c|}{ Arrangement 3} & \multicolumn{4}{|c|}{ Arrangement 4} \\
\hline & Front & Back & Left & Right & Front & Back & Left & Right \\
\hline Cube 1 & Green & White & Blue & Red & Red & White & Blue & Red \\
\hline Cube 2 & Green & White & Red & Green & Blue & White & White & Green \\
\hline Cube 3 & Blue & White & Red & Blue & Green & Green & Red & Blue \\
\hline
\end{tabular}




\begin{tabular}{|c|c|c|c|c|c|c|c|c|}
\hline Cube 4 & Blue & White & Red & Green & Blue & White & Red & Green \\
\hline & \multicolumn{4}{|c|}{ Arrangement 5} & & & & \\
\hline & Front & Back & Left & Right & & & & \\
\hline Cube 1 & Red & White & Blue & Red & & & & \\
\hline Cube 2 & Blue & White & Green & Red & & & & \\
\hline Cube 3 & Blue & White & Red & Blue & & & & \\
\hline Cube 4 & Blue & White & Red & Green & & & & \\
\hline
\end{tabular}

Table 2. Possible arrangements based on front-back-left-right color

\section{STEP 5}

Now we will verify each arrangement one by one.

Initially, none of the arrangements above seem to work, however, we must consider the fact that each cube can rotate in multiple directions while keeping the top and bottom pair unchanged.

For instance, we can simply switch the color between front \& back; left \& right by rotating the cube by 180 degrees. Also, if we rotate the cube 90 degrees, we can switch the color between front \& left; back \& right. We can also perform the combination of both 90 and 180 degree rotation.

After the verification process, we can relocate the arrangement \#3 as in table 3 , which is the solution for the problem.

\begin{tabular}{|c|c|c|c|c|}
\hline & \multicolumn{4}{|c|}{ Arrangement 3} \\
\hline & Front & Back & Left & Right \\
\hline Cube 1 & Green & White & Red & Blue \\
\hline Cube 2 & Red & Green & White & Green \\
\hline Cube 3 & Blue & Red & Blue & White \\
\hline Cube 4 & White & blue & Green & Red \\
\hline
\end{tabular}

Table 3. Relocated arrangement 3

We can easily eliminate non-working arrangement by looking at only 2 columns at a time (either front \& back or left \& right).

First, try to switch the colors in such a way that there are 2 reds, 2 blues, 2 whites, and 2 greens within 2 columns because that is what we are trying to achieve eventually. After a few switch-overs, we can easily notice that certain arrangements are impossible to achieve that allocation, meaning they are not the solution. Then simply move on to the next arrangement and continue the verification process.

Once we find the working arrangement (in this case arrangement\#3), we no longer need to verify remainingarrangements because Instant Insanity has unique solution. 


\section{DIFFERENT EXAMPLE}

In this example as in figure 5, there is no solution for the Instant Insanity problem.

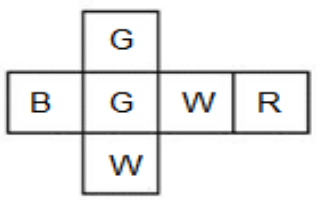

2

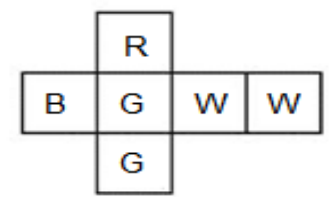

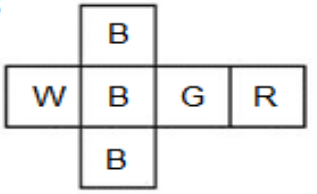

4

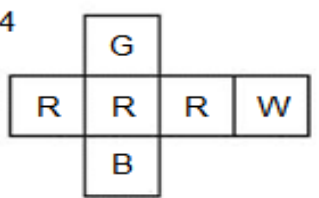

Fig 5.Opened \& flattened representation

\section{STEP 1}

Red: 6

Blue: 6

Green: 6

White: 6

\section{STEP 2}

The top and bottom must consist of:

2 Reds $(6-4=2)$

2 Blues $(6-4=2)$

2 Greens (6-4 = 2)

2 Whites $(6-4=2)$

\section{STEP 3}

\begin{tabular}{|c|c|c|c|c|}
\hline & T-B Pairs & Arrangement 1 & Arrangement 2 & Arrangement 3 \\
\hline \multirow{3}{*}{ Cube 1} & $G-W$ & $\sqrt{ }$ & $\sqrt{ }$ & \\
\hline & $B-W$ & & & \\
\hline & $G-R$ & & & $\sqrt{ }$ \\
\hline \multirow{3}{*}{ Cube 2} & $R-G$ & $\sqrt{ }$ & & \\
\hline & $B-W$ & & & \\
\hline & $G-W$ & & $\sqrt{ }$ & $\sqrt{ }$ \\
\hline \multirow{3}{*}{ Cube 3} & $B-B$ & $\sqrt{ }$ & $\sqrt{ }$ & $\sqrt{ }$ \\
\hline & $W-G$ & & & \\
\hline & $B-R$ & & & \\
\hline \multirow{3}{*}{ Cube 4} & $G-B$ & & & \\
\hline & $R-R$ & & $\sqrt{ }$ & \\
\hline & $R-W$ & $\sqrt{ }$ & & $\sqrt{ }$ \\
\hline
\end{tabular}

Table 4. Possible arrangement based on color pairs

\section{STEP 4}




\begin{tabular}{|c|c|c|c|c|c|c|c|c|}
\hline & \multicolumn{4}{|c|}{ Arrangement 1} & \multicolumn{4}{|c|}{ Arrangement 2} \\
\hline & Front & Back & Left & Right & Front & Back & Left & Right \\
\hline Cube 1 & Green & Red & Blue & White & Green & Red & Blue & White \\
\hline Cube 2 & Green & White & Blue & White & White & Blue & Green & Red \\
\hline Cube 3 & Blue & Red & White & Green & Blue & Red & White & Green \\
\hline \multirow[t]{3}{*}{ Cube 4} & Red & Red & Blue & Green & Red & White & Blue & Green \\
\hline & \multicolumn{4}{|c|}{ Arrangement 3} & & & & \\
\hline & Front & Back & Left & Right & & & & \\
\hline Cube 1 & White & Blue & White & Green & & & & \\
\hline Cube 2 & White & Blue & Green & Red & & & & \\
\hline Cube 3 & Blue & Red & White & Green & & & & \\
\hline Cube 4 & Red & Red & Blue & Green & & & & \\
\hline
\end{tabular}

Table 5. Possible arrangements based on front-back-left-right color

\section{STEP 5}

After verification, none of the arrangements above provide the solution. Therefore, there is no solution.

\section{COMPUTER PROGRAM}

Weused a PerlprogramminglanguagetoimplementthenewapproachforthelnstantInsanity.

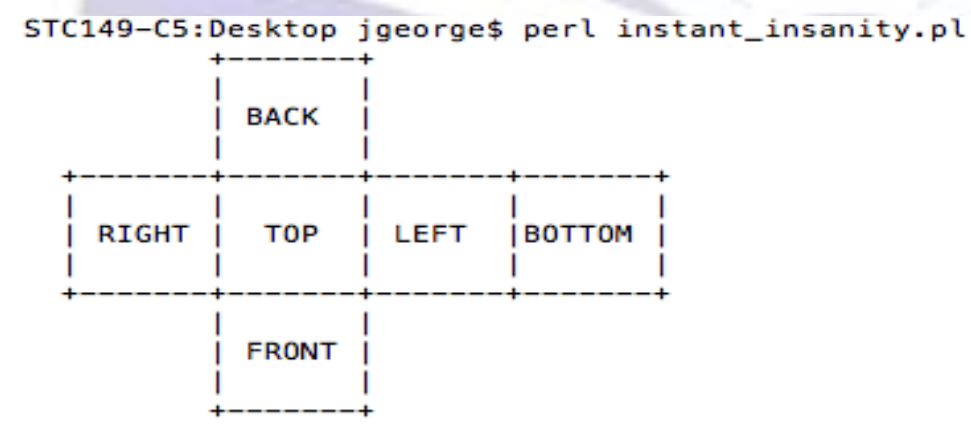

Whenenteringthecolorsenteronlythefirstlettercorrespondingtoeachcolorandintheorderofleft,front, right, back, top, andbottom. $\mathrm{R} / \mathrm{r}$ : red

B/b: blue

G/g: green

W/w: white 
Enterinthecolorsforcube 1:

rbwrgw

Enterinthecolorsforcube 2:

ggwrbw

Enterinthecolorsforcube 3:

brwbgg

Enterinthecolorforcube 4:

grrrbw

ThetopandbottommustconsistofthismanyReds: 3

ThetopandbottommustconsistofthismanyGreens: 2

ThetopandbottommustconsistofthismanyWhites: 2

ThetopandbottommustconsistofthismanyBlues: 1

Found 8 solutions (1 uniquesolution)

$* * * * * * * \quad * * * * * * *$

gbwrbwrg

rggwggwr

bwrbwrbb

wrbgrbgw

$\star \star \star \star * \star * *$

rwbggrwb

wggrrwgg

brwbbbrw

\section{REFERENCES}

[1] Goodaire, E. G., andParmenter, M. M. 2002.DiscreteMathematicswithGraphTheory, Prentice Hall, NewJersy.

[2]Garrido, E.P.B.G. 2013.PuzzleLocuralnstantanea (InstantInsanity) (onlineretrievedfrom: http://divulgamat2.ehu.es/divulgamat15/index.php?option=com_content\& view=article\&id=13498\&directory=67). 